\title{
Aperiodic Sampled-Data Control for Chaotic System Based on Takagi-Sugeno Fuzzy Model
}

\author{
Minjie Zheng (D), Shenhua Yang ${ }^{D}$, and Lina Li \\ Navigation College, Jimei University, Jimei, Xiamen 361021, China \\ Correspondence should be addressed to Shenhua Yang; yangshh@163.com
}

Received 29 July 2020; Revised 9 December 2020; Accepted 16 April 2021; Published 27 April 2021

Academic Editor: Quanxin Zhu

Copyright ( $\odot 2021$ Minjie Zheng et al. This is an open access article distributed under the Creative Commons Attribution License, which permits unrestricted use, distribution, and reproduction in any medium, provided the original work is properly cited.

\begin{abstract}
This paper investigates the aperiodic sampled-data control for a chaotic system. Firstly, Takagi-Sugeno (T-S) fuzzy models for the chaotic systems are established. The lower and upper bounds of the sampling period are taken into consideration. Then, the criteria for mean square exponential stability analysis and aperiodic sampled-data controller synthesis are provided by means of linear matrix inequalities. And the real sampling patterns can be fully captured by constructing suitable Lyapunov functions. Finally, an illustrative example shows that the proposed method is effective to guarantee that the system's states are stable with aperiodic sampled data.
\end{abstract}

\section{Introduction}

Recently, the chaotic system has gradually become one of the hot topics in the field of nonlinear system. The chaotic system has wide application in several areas such as information processing, chemical reaction, power conversion, secure communication biological system, and other aspects. Thus, the control problem for the chaotic system has attracted considerable attention (see $[1,2])$. Among these references, fuzzy control is an effective method to deal with chaotic systems, especially when the plant's knowledge is incomplete or the action cannot be accurately controlled. So far, many scholars have focused on the fuzzy chaotic systems (see [3-8]), and many methods have been developed, such as robust control [9], adaptive control [10], pulse control [11], and sliding mode control [12].

On the other hand, the chaotic system can be described with T-S fuzzy models (TSFM) [13-15], which can be analyzed by using mature linear system theory. Recently, considerable references have been reported about chaotic system with TSFM. In [16], the issue about asymptotic stability for the chaotic system with TSFM is studied, and an impulsive controller is designed. In [17], an adaptive synchronization method for the chaotic system with TSFM is proposed for solving the issue of parameter mismatch. In
[18], the predictive control and synchronization for the chaotic system with TSFM is discussed. And the identical satellite systems are synchronized by the predictive control technique.

In the past few years, the sampled-data system has become an important topic because modern control systems widely used the digital computers to control continuoustime systems (see [19-21]). Compared with the analog controller, the digital controller has better reliability, lower installation, and easier maintenance, which are the advantages of the systems. The outstanding feature of the systems is the coexistence of continuous signal and discrete signal, which is difficult to be analyzed and designed. Until now, the system has attracted much attention of scholars, and considerable results have been reported for that system (see [22-25]). Besides, several sampled-data control approaches such as lifting technology and input delay approach have been used in the systems including many real systems such as the near-space hypersonic vehicles, autonomous airships, unmanned marine vehicles, and so on. Recently, the fuzzy sampled-data chaotic system has attracted considerable attention [26]. In [27], by adaptive event-triggered scheme, the issue about fault-tolerant synchronization for chaotic system is discussed. In [28], a new Lyapunov-Krasovskii function (LKF) method is introduced for the chaotic systems 
with TSFM, and a sampled-data controller is produced to obtain a long sampling period. In [29], the exponential stabilization problem of the fuzzy semi-Markov chaotic system is discussed by establishing a new zero-value equation. In [30], the stochastic sampled-data controllers for the chaotic system are designed, and an improved LKF is constructed to fully exploit the sampling characteristics.

It is noted that the sampling periods of the existing references for the chaotic system are ideally assumed to be constant. However, the sampling period is aperiodic due to the aging of sensors and the interference of noise environment. Hence, to consider nonperiodicity sampling for designing sampled-data controller is practically significant. Besides, in these papers, the lower bound of the sampling period is often considered to be 0 which will lead to considerable conservatism because the value of the variable period may change in a range. Therefore, the lower bound and upper bound of the sampling period should be both considered. Finally, LKF has room for improving to fully capture the real sampling patterns.

Motivated by the above, in this paper, the issue about aperiodic fuzzy sampled-data control of the chaotic system is discussed. Firstly, TSFM is represented for chaotic systems. Then, both lower and upper bounds of the variable period are taken into consideration. In terms of LMI approach, Lyapunov theorem is involved for the stability analysis which can fully capture the sampling patterns. Then, the designed method of fuzzy sampled-data controller is introduced. Finally, a simulation of a chaotic system is conducted to verify the effectiveness of the given strategy.

Notations: $\operatorname{Sym}\{M\}$ represents $M+M^{T}$. “*” represents the symmetric term of a matrix.

\section{Problem Formulation}

Consider a chaotic system as follows:

$$
\dot{x}(t)=f(x(t), u(t)),
$$

where $x(t) \in R^{n}$ denotes the state vector, $u(t) \in R^{m}$ denotes the input vector, and $f(x(t))$ is a nonlinear function which satisfies $f(0,0)=0$. Based on the TSFM, system (1) can be described as follows.

Mode Rule i: IF $z_{1}(t)$ is $\chi_{\mathrm{il}}$, and $\cdots z_{n}(t)$ is $\chi_{\text {in }}$, THEN

$$
\dot{x}(t)=A_{i} x(t)+B_{i} u(t), \quad i=1,2, \ldots, r,
$$

where $\chi_{i 1}, \chi_{i 2}, \ldots, \chi_{i n}$ denote the fuzzy sets, $r$ denotes the number of rules, $z_{1}(t), z_{2}(t), \ldots, z_{\mathrm{n}}(t)$ represent premise variables, and $A_{i}, B_{i}$ denote the appropriated dimensioned matrices. Similar to [9], fuzzy system (2) is given by

$$
\dot{x}(t)=\sum_{i=1}^{r} \mu_{i}(z(t))\left[A_{i} x(t)+B_{i} u(t)\right]
$$

where

$$
\begin{aligned}
\mu_{i}(z(t)) & =\frac{v_{i}(z(t))}{\sum_{i=1}^{r} v_{i}(z(t))} \geq 0, \\
v_{i}(z(t)) & =\prod_{j=1}^{n} \chi_{i j}\left(z_{j}(t)\right), \\
\sum_{i=1}^{r} \mu_{i}(z(t)) & =1, \quad z(t)=\left[z_{1}(t), z_{2}(t), \ldots, z_{n}(t)\right],
\end{aligned}
$$

with $\chi_{i j}\left(z_{j}(t)\right)$ representing the membership grade of $z_{j}(t)$.

Assume that the state variables of chaotic systems are measured in $0=t_{0}<t_{1}<t_{2}<\cdots<t_{k}<\cdots \lim t_{k}=+\infty$, where $t_{k}, k=1,2, \ldots$ is sampling instant. Moreover, the sampling period is aperiodic and satisfies

$$
d_{1} \leq t_{k+1}-t_{k}=d_{k} \leq d_{2}, \quad \forall k \geq 0, d_{1} \geq 0, d_{2}>0 .
$$

The framework of the sampled-data chaotic system is given in Figure 1.

Then, based on parallel distributed compensation, the fuzzy sampled-data controller is designed. THEN

Controller Rule i: IF $z_{1}(t)$ is $\chi_{\mathrm{i} 1}$, and $\ldots z_{n}(t)$ is $\chi_{\mathrm{in}}$,

$$
u(t)=K_{i} x\left(t_{k}\right), \quad t_{k} \leq t<t_{k+1},
$$

where $K$ is a controller matrix. Then, the overall fuzzy model is represented as follows:

$$
u(t)=\sum_{j=1}^{r} \mu_{j}(z(t)) K_{j} x\left(t_{k}\right), \quad t_{k} \leq t<t_{k+1}, k=0,1,2, \ldots
$$

Substituting (7) into equation (3), we obtain

$$
\dot{x}(t)=\sum_{i=1}^{r} \sum_{j=1}^{r} \mu_{i}(z(t)) \mu_{j}(z(t))\left[A_{i} x(t)+B_{i} K_{j} x\left(t_{k}\right)\right] .
$$

The paper's purpose is designing a fuzzy sampled-data controller (FSDC) to satisfy that

(1) System (8) is mean square exponentially stable.

(2) A longer sampling period is achieved.

\section{Main Results}

In this section, the sufficient stability criteria for system (8) are exhibited by establishing LKF firstly. Then, the FSDC will be provided to analyze the stability criteria.

Theorem 1. For scales $\varepsilon_{1}, \varepsilon_{2}$ and $d_{2}>d_{1} \geq 0$, system (8) is mean square exponentially stable, if there exist matrices

$$
\begin{gathered}
Y_{11}, Y_{22}, M, P>0,\left[\begin{array}{cc}
Q_{11} & Q_{12} \\
* & Q_{22}
\end{array}\right]>0, \text { such that } \\
\widetilde{\Psi}_{1}^{i j}(d)=\Psi_{1}^{i j}+d e^{-2 \lambda d_{2}} I_{3} Q_{22} I_{3}^{T}+d \Upsilon_{1}<0, \\
\widetilde{\Psi}_{2}^{i j}(d)=\left[\begin{array}{cc}
\Psi_{1}^{i j} & -d e^{-2 \lambda d_{2}} \Psi_{3} \\
* & -d e^{-2 \lambda d_{2}} Q_{11}
\end{array}\right]<0, \quad d \in\left\{d_{1}, d_{2}\right\},
\end{gathered}
$$




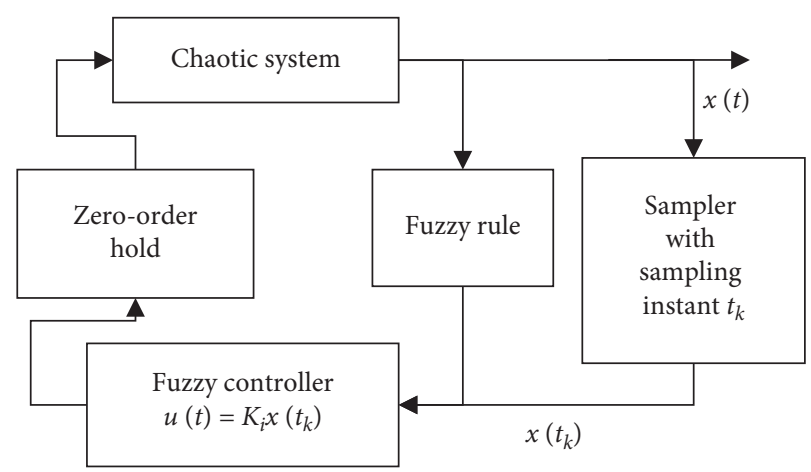

FIgURE 1: The framework of the sampled-data chaotic system.

$$
\Theta=\left[\begin{array}{cc}
P+d_{2}\left(Y_{11}+Y_{11}^{T}\right) & -d_{2}\left(Y_{11}+Y_{22}\right) \\
* & d_{2}\left(Y_{22}+Y_{22}^{T}\right)
\end{array}\right]>0,
$$

$$
\begin{aligned}
\Psi_{1}^{i j}= & \operatorname{Sym}\left\{I_{1} P I_{2}^{T}+\lambda I_{1} P I_{1}^{T}-I_{1} Y_{11} I_{1}^{T}+I_{1} Y_{11} I_{3}^{T}+I_{1} Y_{22} I_{3}^{T}-\varepsilon_{1} I_{1} M A_{i} I_{1}^{T}-I_{1} \bar{A}_{i}^{T} M^{T} I_{2}^{T}-\varepsilon_{1} I_{1} M \bar{B}_{i} \theta K_{j} I_{3}^{T}\right. \\
& \left.+I_{2} M I_{2}^{T}-I_{3} Y_{22} I_{3}^{T}+\varepsilon_{1} I_{1} M I_{2}^{T}-\varepsilon_{1} I_{1} \bar{A}_{i}^{T} M^{T} I_{3}^{T}-I_{2} M \bar{B}_{i} \theta K_{j} I_{3}^{T}+\varepsilon_{2} I_{2} M^{T} I_{3}^{T}-\varepsilon_{1} I_{3} M \bar{B}_{i} \theta K_{j} I_{3}^{T}\right\}+I-\mathrm{d} e^{-2 \lambda d_{2}} I_{3} Q_{22} I_{3}^{T}, \\
\Upsilon_{1}= & \operatorname{Sym}\left\{I_{1}\left(Y_{11}+Y_{11}^{T}\right) I_{2}^{T}-I_{2}\left(Y_{11}+Y_{22}\right) I_{3}^{T}+I_{2} Q_{11} I_{2}^{T}\right\}+I_{2} Q_{12} I_{3}^{T}+\lambda I_{1}\left(Y_{11}+Y_{11}^{T}\right) I_{1}^{T} \\
& \left.-\lambda I_{1}\left(Y_{11}+Y_{22}\right) I_{3}^{T}+I_{3} Q_{22} I_{3}^{T}+\lambda I_{3}\left(Y_{22}+Y_{22}^{T}\right) I_{3}^{T}\right\} \\
\Psi_{3}= & {\left[\begin{array}{lll}
0 & 0 & Q_{12}
\end{array}\right]^{T} . }
\end{aligned}
$$

Proof. The novel LKF is proposed:

$$
\begin{aligned}
& V(t)=\sum_{i=1}^{3} V_{i}(t), \quad t \in\left[t_{k}, t_{k+1}\right), \\
& V_{1}(t)=x(t)^{T} P x(t), \\
& V_{2}(t)=h_{2}(t) \int_{t_{k}}^{t} e^{2 \lambda(s-t)}\left[\begin{array}{c}
\dot{x}(s) \\
x\left(t_{k}\right)
\end{array}\right]^{T}\left[\begin{array}{cc}
Q_{11} & Q_{12} \\
* & Q_{22}
\end{array}\right]\left[\begin{array}{c}
\dot{x}(s) \\
x\left(t_{k}\right)
\end{array}\right] \mathrm{d} s, \\
& V_{3}(t)=h_{2}(t)\left[\begin{array}{c}
x(t) \\
x\left(t_{k}\right)
\end{array}\right]^{T}\left[\begin{array}{cc}
Y_{11}+Y_{11}^{T} & -Y_{11}-Y_{22} \\
* & Y_{22}+Y_{22}^{T}
\end{array}\right]\left[\begin{array}{c}
x(t) \\
x\left(t_{k}\right)
\end{array}\right] .
\end{aligned}
$$

It is noted that $\lim _{t \longrightarrow t_{k}^{-}} V_{i}(t)=\lim _{t \longrightarrow t_{k}^{+}} V_{i}(t)=V_{i}(t)=0$, $i=2,3$, and hence $V(t)$ is continuous on $[0, \infty)$. To reduce the conservatism, the matrix in $V_{3}(t)$ is free; then, according to condition (11), it can be obtained that

$$
\begin{aligned}
V_{1}(t)+V_{3}(t) & =\frac{d_{2}-h_{2}(t)}{d_{2}} x^{T}(t) P x(t)+\frac{h_{2}(t)}{d_{2}} x^{T}(t) P x(t)+\frac{h_{2}(t)}{d_{2}} d_{2}\left[\begin{array}{c}
x(t) \\
x\left(t_{k}\right)
\end{array}\right]^{T}\left[\begin{array}{cc}
Y_{11}+Y_{11}^{T} & -Y_{11}-Y_{22} \\
* & Y_{22}+Y_{22}^{T}
\end{array}\right]\left[\begin{array}{c}
\dot{x}(t) \\
x\left(t_{k}\right)
\end{array}\right] \\
& =\frac{d_{2}-h_{2}(t)}{d_{2}} x^{T}(t) P x(t)+\frac{h_{2}(t)}{d_{2}}\left[\begin{array}{c}
x(t) \\
x\left(t_{k}\right)
\end{array}\right]^{T}\left[\begin{array}{c}
\dot{x}(t) \\
x\left(t_{k}\right)
\end{array}\right] \geq 0 .
\end{aligned}
$$


Taking the derivative of $V(t)$, the following can be obtained:

$$
\begin{aligned}
\dot{V}_{1}(t)+2 \lambda V_{1}(t) & =2 x^{T}(t) P \dot{x}(t)+2 \lambda x^{T}(t) P x(t) \\
\dot{V}_{2}(t)+2 \lambda V_{2}(t)= & \left.h_{2}(t)\left[\begin{array}{c}
\dot{x}(t) \\
x\left(t_{k}\right)
\end{array}\right]^{T}\left[\begin{array}{cc}
Q_{11} & Q_{12} \\
* & Q_{22}
\end{array}\right]\left[\begin{array}{c}
\dot{x}(t) \\
x\left(t_{k}\right)
\end{array}\right]-e^{-2 \lambda d_{2}}\right]_{t_{k}}^{t}\left[\begin{array}{c}
\dot{x}(s) \\
x\left(t_{k}\right)
\end{array}\right]^{T}\left[\begin{array}{cc}
Q_{11} & Q_{12} \\
* & Q_{22}
\end{array}\right]\left[\begin{array}{c}
\dot{x}(s) \\
x\left(t_{k}\right)
\end{array}\right] d s \\
\dot{V}_{3}(t)+2 \lambda V_{3}(t)= & 2 h_{2}(t)\left[\begin{array}{c}
x(t) \\
x\left(t_{k}\right)
\end{array}\right]^{T}\left[\begin{array}{cc}
Y_{11}+Y_{11}^{T} & -Y_{11}-Y_{22} \\
* & Y_{22}+Y_{22}^{T}
\end{array}\right]\left[\begin{array}{c}
\dot{x}(t) \\
0
\end{array}\right]-\left[\begin{array}{c}
x(t) \\
x\left(t_{k}\right)
\end{array}\right]^{T}\left[\begin{array}{cc}
Y_{11}+Y_{11}^{T} & -Y_{11}-Y_{22} \\
* & Y_{22}+Y_{22}^{T}
\end{array}\right]\left[\begin{array}{c}
x(t) \\
x\left(t_{k}\right)
\end{array}\right] \\
& +2 \lambda h_{2}(t)\left[\begin{array}{c}
x(t) \\
x\left(t_{k}\right)
\end{array}\right]^{T}\left[\begin{array}{cc}
Y_{11}+Y_{11}^{T} & -Y_{11}-Y_{22} \\
* & Y_{22}+Y_{22}^{T}
\end{array}\right]\left[\begin{array}{c}
x(t) \\
x\left(t_{k}\right)
\end{array}\right] .
\end{aligned}
$$

For any free matrix $M$ and scalars $\varepsilon_{1}, \varepsilon_{2}$, we can obtain the equation as follows:

$$
2 \sum_{i=1}^{3} \sum_{j=1}^{3} \mu_{i}(z(t)) \mu_{j}(z(t))\left[\varepsilon_{1} x^{T}(t) M+\dot{x}^{T}(t) M+\varepsilon_{2} x^{T}\left(t_{k}\right) M\right] \times\left[\dot{x}(t)-\left(\bar{A}_{i} x(t)+\bar{B}_{i} K_{j} x\left(t_{k}\right)\right)\right]=0 .
$$

Then, combining (15) and (16), we have

$$
\dot{V}(t)+2 \lambda V(t)=2 \sum_{i=1}^{3} \sum_{j=1}^{3} \mu_{i}(z(t)) \mu_{j}(z(t))\left[\frac{h_{2}(t)}{\mathrm{d}_{k}} \zeta^{T}(t) \widetilde{\Psi}_{1}^{i j}\left(\mathrm{~d}_{k}\right) \zeta(t) \frac{1}{\mathrm{~d}_{k}} \int_{t_{k}}^{t} \zeta^{T}(t, s) \widetilde{\Psi}_{2}^{i j}\left(\mathrm{~d}_{k}\right) \zeta(t, s) \mathrm{d} s\right]
$$

where

$$
\begin{aligned}
\zeta(t) & =\left[\begin{array}{lll}
x^{T}(t) & \dot{x}^{T}(t) & x^{T}\left(t_{k}\right)
\end{array}\right]^{T}, \\
\zeta(t, s) & =\left[\begin{array}{llll}
x^{T}(t) & \dot{x}^{T}(t) & x^{T}\left(t_{k}\right) & \dot{x}^{T}(s)
\end{array}\right]^{T} .
\end{aligned}
$$

From (9) and (10), the following can be obtained:

$$
\begin{aligned}
& \widetilde{\Psi}_{1}^{i j}\left(d_{k}\right)=\frac{d_{k}-d_{1}}{d_{2}-d_{1}} \widetilde{\Psi}_{1}^{i j}\left(d_{2}\right)+\frac{d_{2}-d_{k}}{d_{2}-d_{1}} \widetilde{\Psi}_{1}^{i j}\left(d_{1}\right)<0, \\
& \widetilde{\Psi}_{2}^{i j}\left(d_{k}\right)=\frac{d_{k}-d_{1}}{d_{2}-d_{1}} \widetilde{\Psi}_{2}^{i j}\left(d_{2}\right)+\frac{d_{2}-d_{k}}{d_{2}-d_{1}} \widetilde{\Psi}_{2}^{i j}\left(d_{1}\right)<0,
\end{aligned}
$$

which implies that

$$
\dot{V}(t)+2 \lambda V(t)<0,
$$

and then we have

$$
\begin{aligned}
\lambda_{\min }(P)\left\{\|x(t)\|^{2}\right\} & \leq\{V(t)\}<e^{-2 \lambda\left(t-t_{k}\right)}\left\{V\left(t_{k}\right)\right\} \\
& <e^{-2 \lambda\left(t-t_{k-1}\right)}\left\{V\left(t_{k-1}\right)\right\}<\cdots<e^{-2 \lambda t}\{V(0)\} \\
& \leq \lambda_{\max }(P) e^{-2 \lambda t}\left\|x\left(t_{0}\right)\right\|^{2} .
\end{aligned}
$$

Then

$$
\{\|x(t)\|\} \leq\left(\frac{\sqrt{\lambda_{\max }(P)}}{\sqrt{\lambda_{\min }(P)}}\right) e^{-\lambda t}\left\|x\left(t_{0}\right)\right\|,
$$

which implies that system (8) is mean square exponentially stable. This completed the proof.

Remark 1 . The mean square exponential stability criteria for system (8) are introduced in Theorem 1. To fully characterize the sampling pattern, a suitable time-dependent LKF (11) is constructed and a novel quadratic function $V_{3}(t)$ is added in LKF (11).

Remark 2. For the purpose of reducing the conservatism, more relaxed constraint matrices are introduced in LKF (13). The matrices are not required to be positive, which can effectively reduce conservatism.

Furthermore, the sampled-data controller (7) will be designed for stabilizing system (8) based on the following theorems.

Theorem 2. For scales $\varepsilon_{1}, \varepsilon_{2}$ and $d_{2}>d_{1} \geq 0$, system (8) is mean square exponentially stable, if there exist matrices $\bar{Y}_{11}, \bar{Y}_{22}, \bar{M}, \bar{P}>0,\left[\begin{array}{cc}\bar{Q}_{11} & \bar{Q}_{12} \\ * & \bar{Q}_{22}\end{array}\right]>0$, satisfying 


$$
\begin{aligned}
& \overline{\widetilde{\Psi}}_{1}^{i j}(d)=\left[\begin{array}{cc}
\bar{\Psi}_{1}^{i j}+d e^{-2 \lambda d_{2}} I_{3} \bar{Q}_{22} I_{3}^{T}+d \bar{\Upsilon}_{1} & \bar{\Psi}_{4} \\
* & -I
\end{array}\right]<0, \\
& \bar{\Psi}_{2}^{i j}(d)=\left[\begin{array}{ccc}
\bar{\Psi}_{1}^{i j} & -d e^{-2 \lambda d_{2}} \bar{\Psi}_{3} & \bar{\Psi}_{4} \\
* & -d e^{-2 \lambda d_{2}} \bar{Q}_{11} & 0 \\
* & * & -I
\end{array}\right]<0, \quad d \in\left\{d_{1}, d_{2}\right\},
\end{aligned}
$$

$$
\bar{\Theta}=\left[\begin{array}{cc}
\bar{P}+d_{2}\left(\bar{Y}_{11}+\bar{Y}_{11}^{T}\right) & -d_{2}\left(\bar{Y}_{11}+\bar{Y}_{22}\right) \\
* & d_{2}\left(\bar{Y}_{22}+\bar{Y}_{22}^{T}\right)
\end{array}\right]>0,
$$

where

$$
\begin{aligned}
\bar{\Psi}_{1}^{i j}= & \operatorname{Sym}\left\{I_{1} \bar{P} I_{2}^{T}+\lambda I_{1} \bar{P} I_{1}^{T}-I_{1} \bar{Y}_{11} I_{1}^{T}+I_{1} \bar{Y}_{11} I_{3}^{T}+I_{1} \bar{Y}_{22} I_{3}^{T}-\varepsilon_{1} I_{1} \bar{A}_{i} \bar{M} I_{1}^{T}-I_{1} \bar{M}_{i}^{T} I_{2}^{T}\right. \\
& \left.-\varepsilon_{1} I_{1} \bar{B}_{i} \theta \bar{K}_{j} I_{3}^{T}+I_{2} \bar{M} I_{2}^{T}-I_{3} \bar{Y}_{22} I_{3}^{T}+\varepsilon_{1} I_{1} \bar{M} I_{2}^{T}-\varepsilon_{1} I_{1} \bar{M}_{i}^{T} I_{3}^{T}-I_{2} \bar{B}_{i} \theta \bar{K}_{j} I_{3}^{T}+\varepsilon_{2} I_{2} \bar{M}^{T} I_{3}^{T}-\varepsilon_{1} I_{3} \bar{B}_{i} \theta \bar{K}_{j} I_{3}^{T}\right\}-\mathrm{d} e^{-2 \lambda d_{2}} I_{3} \bar{Q}_{22} I_{3}^{T} \\
\bar{\Upsilon}_{1}= & \operatorname{Sym}_{1}\left\{\left(\bar{Y}_{11}+\bar{Y}_{11}^{T}\right) I_{2}^{T}-I_{2}\left(\bar{Y}_{11}+\bar{Y}_{22}\right) I_{3}^{T}+I_{2} \bar{Q}_{11} I_{2}^{T}+I_{2} \bar{Q}_{12} I_{3}^{T}+\lambda I_{1}\left(\bar{Y}_{11}+\bar{Y}_{11}^{T}\right) I_{1}^{T}\right. \\
& \left.-\lambda I_{1}\left(\bar{Y}_{11}+\bar{Y}_{22}\right) I_{3}^{T}+I_{3} \bar{Q}_{22} I_{3}^{T}+\lambda I_{3}\left(\bar{Y}_{22}+\bar{Y}_{22}^{T}\right) I_{3}^{T}\right\} \\
\bar{\Psi}_{3}= & {\left[\begin{array}{lll}
0 & 0 & \bar{Q}_{12}
\end{array}\right]^{T} } \\
\bar{\Psi}_{4}= & {\left[\begin{array}{lll}
\bar{M}^{T} & 0 & 0
\end{array}\right]^{T} . }
\end{aligned}
$$

Then, the controller gain matrix Kcan be obtained as follows:

$$
K=\overline{K M}^{-T}
$$

Proof. Let

$$
\begin{aligned}
& \chi_{1}=\bar{M}, \\
& \chi_{2}=\operatorname{diag}\{\bar{M}, \bar{M}\}, \\
& \chi_{3}=\operatorname{diag}\left\{\chi_{2}, \bar{M}, I\right\} .
\end{aligned}
$$

Define

$$
\begin{aligned}
\bar{M} & =M^{-1}, \\
\bar{P} & =\bar{M} P \bar{M}^{T}, \\
Q_{11} & =\bar{M} Q_{11} \bar{M}^{T}, \\
Q_{12} & =\bar{M} Q_{12} \bar{M}^{T}, \\
Q_{22} & =\bar{M} Q_{22} \bar{M}^{T}, \\
Y_{11} & =\bar{M} Y_{11} \bar{M}^{T}, \\
Y_{22} & =\bar{M} Y_{22} \bar{M}^{T}, \\
\bar{K} & =K \bar{M}^{T} .
\end{aligned}
$$

Pre- and post-multiply (9)-(11) by $\chi_{1}, \chi_{2}$, and $\chi_{3}$ respectively; then, according to Schur complement, (23)-(25) can be obtained. This completed the proof.

Remark 3. Note that the lower and upper bounds of the variable period are both considered in Theorem 1. If $d_{1}=d_{2}=d$, the periodic sampled-data issue for chaotic system can be solved, and the sampling period will reduce to be constant like [31], which have strict limitations on the lower bound of the delay and it will lead to considerable conservatism. So, the result in Theorem 1 covers [31] as a special case and has more significance than [31].

\section{Numerical Examples}

In the section, a simulation example for a chaotic system will be used to verify the effectiveness of given methods.

Consider a chaotic system as follows [15]:

$$
\left\{\begin{array}{l}
\dot{x}_{1}(t)=-a x_{1}(t)+a x_{2}(t), \\
\dot{x}_{2}(t)=c x_{1}(t)-x_{2}(t)-x_{1}(t) x_{3}(t)+u_{1}(t), \\
\dot{x}_{3}(t)=x_{1}(t) x_{2}(t)-b x_{3}(t)+u_{2}(t),
\end{array}\right.
$$

where

$$
\begin{aligned}
& a=10, \\
& b=\frac{8}{3}, \\
& c=28 .
\end{aligned}
$$

Assume that $x_{1}(t) \in\left[m_{1}, m_{2}\right], \dot{x}_{1}(t) \in[-5,5]$. According to [15], system (30) is represented with TSFM as follows.

Mode rule 1: IF $x_{1}(t)$ is $\omega_{1}$, THEN

$$
\dot{x}(t)=A_{1} x(t)+B_{1} u(t) .
$$

Mode rule 2: IF $x_{1}(t)$ is $\omega_{2}$, THEN

$$
\dot{x}(t)=A_{2} x(t)+B_{2} u(t)
$$

where 


$$
\begin{aligned}
& A_{1}=\left[\begin{array}{ccc}
-a & a & 0 \\
c & -1 & m_{1} \\
0 & -m_{1} & -b
\end{array}\right], \\
& A_{2}=\left[\begin{array}{ccc}
-a & a & 0 \\
c & -1 & m_{2} \\
0 & -m_{2} & -b
\end{array}\right], \\
& B_{1}=B_{2}=\left[\begin{array}{ll}
0 & 0 \\
1 & 0 \\
0 & 1
\end{array}\right] .
\end{aligned}
$$

Then, the membership functions are

$$
\begin{aligned}
\mu_{1}(x(t)) & =\frac{x_{1}(t)-m_{1}}{m_{2}-m_{1}}, \\
\mu_{2}\left(x_{1}(t)\right) & =\frac{-x_{1}(t)-m_{2}}{m_{2}-m_{1}},
\end{aligned}
$$

and the trajectories of system (30) are exhibited in Figure 2.

Let $m_{1}=-25, m_{2}=25$. Firstly, we compare the results in Theorem 2 with the references which use different sampleddata control methods for chaotic systems. We suppose that $\tau_{m}=\tau_{M}$ and choose the same parameters; then, the maximum sampling period $\tau_{M}$ obtained by different references is shown in Table 1. It is obvious that Theorem 2 has longer sampling period than that in $[15,28,31]$, which improves about $131.2 \%, 68.1 \%$, and $19.35 \%$, respectively. Hence, the result in the paper can reduce the conservatism to some extent. Besides, the lower and upper bounds of the sampling period are both considered in the paper, which has more significance than the previous work.

Then, we verify the effectiveness of the given method. The initial state is $x(t)=\left[\begin{array}{lll}-5 & 3 & 6\end{array}\right]$. Assuming the sampling interval $\tau_{m}=0$.6and $\varepsilon_{1}=1, \varepsilon_{2}=0.01$, then the maximum upper bound of sampling period is obtained as $\tau_{M}=1.33$ by Theorem 2 . Then, the related matrices by solving conditions (23)-(25) are obtained as

$$
\begin{aligned}
& \bar{K}_{1}=10^{-9} \times\left[\begin{array}{lll}
0.0028 & 0.2369 & 0.0174
\end{array}\right], \\
& \bar{K}_{2}=10^{-9} \times\left[\begin{array}{lll}
0.0028 & 0.2369 & 0.0174
\end{array}\right], \\
& \bar{M}=10^{-9} \times\left[\begin{array}{ccc}
0.3904 & 0.0219 & -0.0005 \\
-0.6132 & 0.1233 & 0.0023 \\
0.0083 & -0.1864 & 0.0007
\end{array}\right] .
\end{aligned}
$$

Then, from (27), we can obtain the controller gain matrix.

$$
\begin{aligned}
& K_{1}=\bar{K}_{1} M^{-T}=\left[\begin{array}{lll}
13.5097 & 8.6778 & 6.0571
\end{array}\right], \\
& K_{2}=\bar{K}_{2} M^{-T}=\left[\begin{array}{lll}
13.5093 & 8.6776 & 6.0569
\end{array}\right] .
\end{aligned}
$$

Under controller (37), the responses of the state are shown in Figures 3-5, which indicate that the states are stable in a short time. It is easy to know that the designed FSDC achieves system's stabilization successfully, and a longer sampling period is obtained.

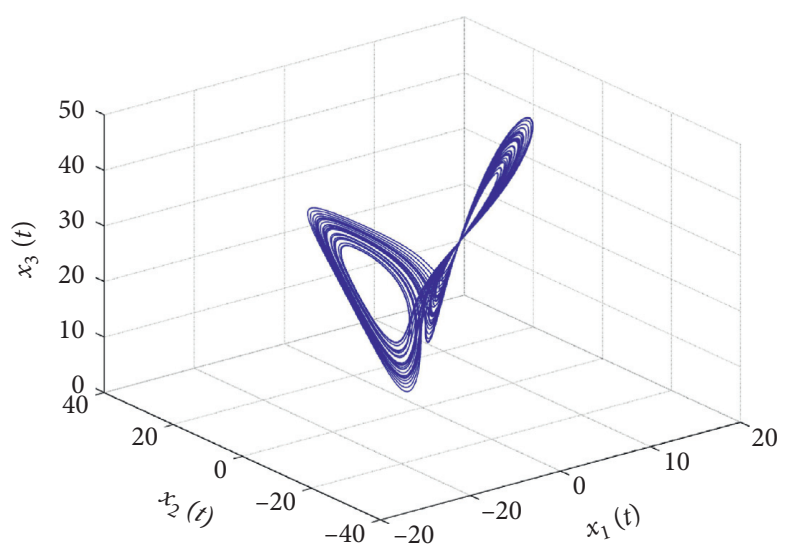

FIgURE 2: Trajectory of the chaotic system.

TABle 1: The maximum upper bound for $\tau_{M}$.

\begin{tabular}{lcccc}
\hline Method & {$[28]$} & {$[31]$} & {$[15]$} & Theorem 2 \\
\hline$\tau_{M}$ & 0.0016 & 0.0022 & 0.0031 & 0.0037 \\
\hline
\end{tabular}

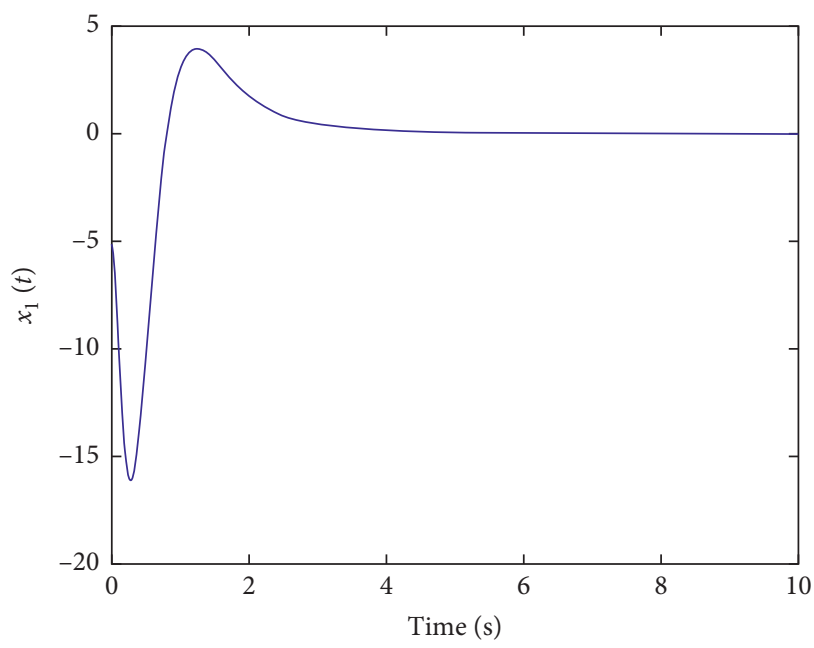

Figure 3: Responses of $x_{1}(t)$.

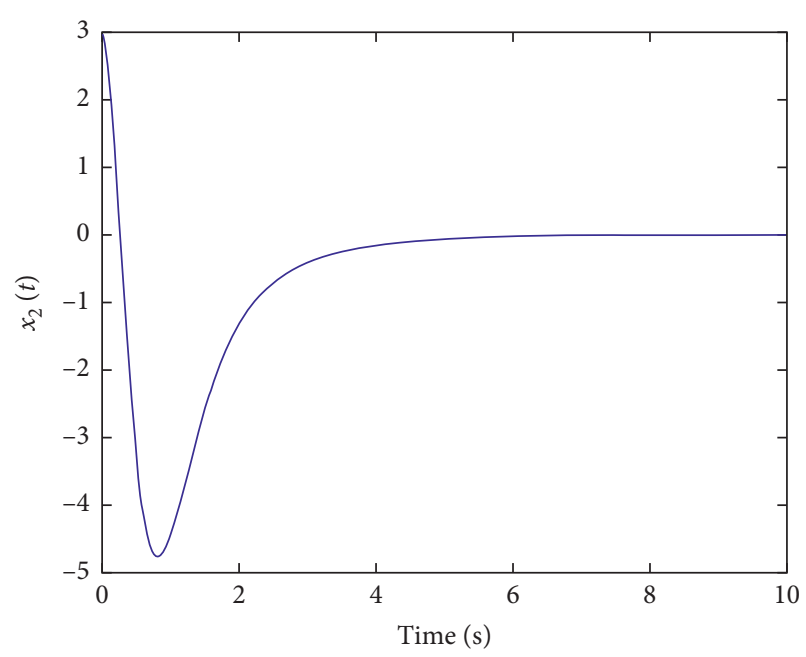

Figure 4: Responses of $x_{2}(t)$. 


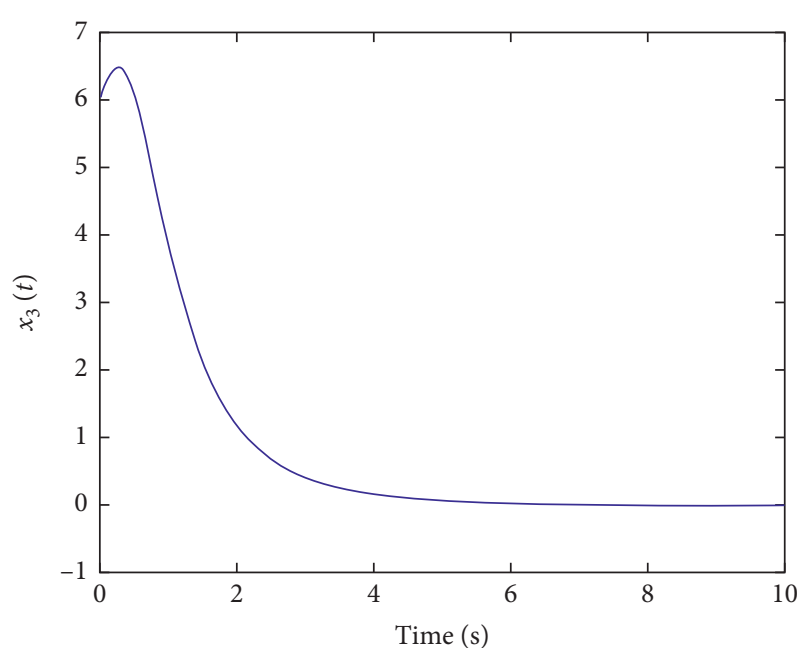

Figure 5: Responses of $x_{3}(t)$.

\section{Conclusions}

This paper discusses the aperiodic sampled-data control problem for a chaotic system with TSFM. And both lower and upper bounds of the sampling period are considered in the paper. Then, the criteria of mean square exponential stability are given. By constructing an appropriate LKF, the sampling patterns are fully captured and less conservative result is obtained. The simulation result is used to verify that the proposed fuzzy aperiodic sampled-data control strategy is effective.

\section{Data Availability}

No data were used to support this study.

\section{Conflicts of Interest}

The authors declare that they have no conflicts of interest.

\section{Acknowledgments}

This study was supported by the National Natural Science Foundation of China (51579114 and 51879119), Natural Science Foundation of Fujian Province (2018J01484 and 2020J01660), and Youth Innovation Foundation of Xiamen (3502Z20206019).

\section{References}

[1] S. Vaidyanathan and S. Sampath, "Anti-synchronization of four-wing chaotic systems via sliding mode control," International Journal of Automation and Computing, vol. 9, no. 3, pp. 274-279, 2012.

[2] D. H. Ji, S. C. Jeong, J. H. Park, and S. C. Won, "Robust adaptive backstepping synchronization for a class of uncertain chaotic systems using fuzzy disturbance observer," Nonlinear Dynamics, vol. 69, no. 3, pp. 1125-1136, 2012.

[3] N. Wang, M. J. Er, M. Han, and M. Han, "Large tanker motion model identification using generalized ellipsoidal basis function-based fuzzy neural networks," IEEE Transactions on Cybernetics, vol. 45, no. 12, pp. 2732-2743, 2015.

[4] R.-E. Precup and M. L. Tomescu, "Stable fuzzy logic control of a general class of chaotic systems," Neural Computing and Applications, vol. 26, no. 3, pp. 541-550, 2015.

[5] W. Xiang, Y. Sun, and H. Liu, "Fuzzy adaptive prescribed performance control for a class of uncertain chaotic systems with unknown control gains," International Journal of Innovative Computing, Information and Control, vol. 12, no. 2, pp. 603-613, 2016.

[6] Z. Liu and R. Guo, "Stabilization of a class of complex chaotic systems by the dynamic feedback control," Complexity, vol. 2020, Article ID 4938149, 10 pages, 2020.

[7] H. Medhaffar, M. Feki, and N. Derbel, "Adaptive fuzzy control for the stabilisation of chaotic systems," International Journal of Automation and Control, vol. 14, no. 2, pp. 115-137, 2020.

[8] H. K. Lam, W.-K. Ling, H. H.-C. Iu, and S. S. H. Ling, "Synchronization of chaotic systems using time-delayed fuzzy state-feedback controller," IEEE Transactions on Circuits and Systems I: Regular Papers, vol. 55, no. 3, pp. 893-903, Apr. 2008.

[9] S.-Y. Li and M. A. B. Hernández, "Robust synchronization of chaotic systems with novel fuzzy rule-based controllers," Information Sciences, vol. 481, pp. 604-615, 2019.

[10] J.-H. Kim, C.-H. Hyun, E. Kim, and M. Park, "Adaptive synchronization of uncertain chaotic systems based on T-S fuzzy model," IEEE Transactions on Fuzzy Systems, vol. 15, no. 3, pp. 359-369, Jun. 2007.

[11] C. Hu, H. Jiang, and Z. Teng, "General impulsive control of chaotic systems based on a TS fuzzy model," Fuzzy Sets and Systems, vol. 174, no. 1, pp. 66-82, Jul. 2011.

[12] S. M. A. Pahnehkolaei, A. Alfi, and J. T. Machado, "Fuzzy logic embedding of fractional order sliding mode and state feedback controllers for synchronization of uncertain fractional chaotic systems," Computational and Applied Mathematics, vol. 39, no. 3, pp. 1-16, 2020.

[13] T. Takagi and M. Sugeno, "Fuzzy identification of systems and its applications to modelling and control," IEEE Transactions on Systems, Man, and Cybernetics, vol. 15, no. 1, pp. 116-132, 1985.

[14] K. Tanaka and H. Wang, Fuzzy Control Systems Design and Analysis: A Linear Matrix Inequality Approach, John Wiely \& Sons, New York, NY, USA, 2001.

[15] Y. Wang, Y. Xia, and P. Zhou, "Fuzzy-model-based sampleddata control of chaotic systems: a fuzzy time-dependent Lyapunov-Krasovskii functional approach," IEEE Transactions on Fuzzy Systems, vol. 25, no. 6, pp. 1672-1684, 2016.

[16] P. Shi, X. Su, and F. Li, "Dissipativity-based filtering for fuzzy switched systems with stochastic perturbation," IEEE Transactions on Automatic Control, vol. 61, no. 6, pp. 16941699, 2016.

[17] J.-H. Kim, C.-W. Park, E. Kim, and M. Park, "Fuzzy adaptive synchronization of uncertain chaotic systems," Physics Letters A, vol. 334, no. 4, pp. 295-305, 2005.

[18] A. Khan and S. Kumar, "T-S fuzzy modeling and predictive control and synchronization of chaotic satellite systems," International Journal of Modelling and Simulation, vol. 39, no. 3, pp. 203-213, 2019.

[19] K. Delchev, G. Boiadjiev, H. Kawasaki, and T. Mouri, "Iterative learning control with sampled-data feedback for robot manipulators," Archives of Control Sciences, vol. 24, no. 3, pp. 299-319, 2014.

[20] S. M. Abedi, "An optimal sample-data holds by using a Biobjective criterion: trade-off between the phase delay and the 
stability robustness," Control Engineering and Applied Informatics, vol. 17, no. 2, pp. 32-42, 2015.

[21] Y. Wang, H. R. Karimi, H.-K. Lam, and H. Shen, "An improved result on exponential stabilization of sampled-data fuzzy systems," IEEE Transactions on Fuzzy Systems, vol. 26, no. 6, pp. 3875-3883, 2018.

[22] E. Fridman, "Robust sampled-data $\$ \mathrm{H} \_$infty $\$$ Control of linear singularly perturbed systems," IEEE Transactions on Automatic Control, vol. 51, no. 3, pp. 470-475, 2006.

[23] H. J. Lee, M. H. Kim, S. Y. Lee, and T. Y. Kim, "Robust sampled-data fuzzy control of nonlinear systems with parametric uncertainties: its application to depth control of autonomous underwater vehicles," International Journal of Control Automation and Systems, vol. 10, no. 6, pp. 11641172, 2012.

[24] Z. G. Wu, P. Shi, and H. Y. Su, "Stochastic synchronization of markovian jump neural networks with time-varying delay using sampled data," IEEE Transactions on Cybernetics, vol. 43, no. 6, pp. 796-1806, 2013.

[25] M. Rubagotti, D. M. Raimondo, A. Ferrara, and L. Magni, "Robust model predictive control with integral sliding mode in continuous-time sampled-data nonlinear systems," IEEE Transactions on Automatic Control, vol. 56, no. 3, pp. 556570, 2011.

[26] Y. Wang, Y. Zhu, H. R. Karimi, and X. Li, "Sampled-data exponential synchronization of chaotic Lur'e systems," IEEE Access, vol. 5, pp. 17834-17840, 2017.

[27] X. Li, D. Ma, X. Xie, and Q. Sun, "Fault-Tolerant synchronization of chaotic systems with fuzzy sampled data controller based on adaptive event-triggered scheme," International Journal of Fuzzy Systems, vol. 1-13, 2020.

[28] Z.-G. Wu, P. Shi, H. Su, and J. Chu, "Sampled-data fuzzy control of chaotic systems based on a T-S fuzzy model," IEEE Transactions on Fuzzy Systems, vol. 22, no. 1, pp. 153-163, 2014.

[29] T. Wu, J. Cao, L. Xiong, and H. Zhang, "New stabilization results for semi-markov chaotic systems with fuzzy sampleddata control," Complexity, vol. 2019, Article ID 7875305, 15 pages, 2019.

[30] N. Gunasekaran and Y. H. Joo, "Stochastic sampled-data controller for T-S fuzzy chaotic systems and its applications," IET Control Theory \& Applications, vol. 13, no. 12, pp. 1834-1843, 2019.

[31] Z. P. Wang and H. N. Wu, "On fuzzy sampled-data control of chaotic systems via a time-dependent Lyapunov functional approach," IEEE Trans. Cybern.vol. 45, no. 4, pp. 819-829, 2015. 